\title{
MODELING PARTICLE HEATING AND CURRENT DRIVE IN FUSION MACHINES: BRIEF OVERVIEW OF ADOPTED TECHNIQUES
}

\author{
D. Van Eester \\ Laboratorium voor Plasmafysica - Laboratoire de Physique des Plasmas \\ EUROfusion Consortium member \\ Koninklijke Militaire School - Ecole Royale Militaire \\ Trilateral Euregio Cluster, Renaissancelaan 30 - B1000 Brussels - Belgium \\ Tel.: (32 2) 4414 134,fax.: (32 2)7352421, e-mail: d.van.eester@fz-juelich.de
}

\begin{abstract}
A brief overview is given of the techniques adopted for modeling plasma heating and current drive as well as the associated particle diffusion. Weaknesses and strengths of each method are highlighted; references are provided for those seeking further information.
\end{abstract}

\section{INTRODUCTION: THE BASIC EQUATIONS}

Plasma heating by virtue of RF (radio frequency) waves can be looked at from two complementary points of view. From the wave's viewpoint plasma heating is a loss process. Solving the relevant wave equation, $\nabla \times \nabla \times \vec{E} / k_{o}^{2}=\mathbf{K} \cdot \vec{E}+i \overrightarrow{J_{a}} / \omega \epsilon_{o}=\vec{E}+i\left[\overrightarrow{J_{a}}+\overrightarrow{J_{p}}\right] / \omega \epsilon_{o}$ reveals where and to which particles the wave energy is lost. Here, $\vec{E}$ is the electric field, $\mathbf{K}$ is the dielectric tensor, $\omega$ is the driver frequency, and $\vec{J}_{a}$ and $\vec{J}_{p}$ are the antenna and plasma current densities. From the particle's point of view, plasma heating is the process of being accelerated or decelerated by an electric field. The net diffusion of particles resulting from this is described by the Fokker-Planck equation, $\frac{\partial F_{o}}{\partial t}=Q+C+S-L$ in which $F_{o}$ is the distribution function, $Q$ is the RF quasilinear diffusion term, $C$ represents the effect of the Coulomb collisions, $S$ is a source and $L$ a loss term. Solving the coupled wave + Fokker-Planck equations involves tackling a 6-dimensional problem. Two - equivalent - approaches have been proposed to achieve this: the trajectory integral technique and the Hamiltonian formalism [1]. The key is to rewrite both equations in terms of shared building blocks describing the interaction of a particular wave component with a guiding center orbit. In absence of an external electric field, the orbits can be expressed in terms of 3 independent constants of the motion $\vec{\Lambda}$ and 3 angles $\vec{\Phi}$ (which - think of Hamiltonian action-angle variables - vary linearly with time and describe the rapid oscillatory aspects of the motion i.e. the poloidal bounce, the toroidal drift and the cyclotron motions). When wave power is injected, the $\vec{\Lambda}$ are no longer constant as a function of time. The coefficients of the Fokker-Planck equation require removing all fast time scale effects i.e. filtering out all oscillatory motion. Evaluating the dielectric response in the wave equation involves integrating over the velocity space coordinates. This can either be done recasting the wave equation in a form directly amenable for nonlocal treatment [2] or it can be done by writing down a local expression for the dielectric tensor (see e.g. [3, 4]). Since the wave and Fokker-Planck equations describe 2 aspects of the same physics, they should be solved as a coupled system of equations (see e.g. [5] and the references therein). Because of the complexity involved, they are, however, solved separately in most applications.

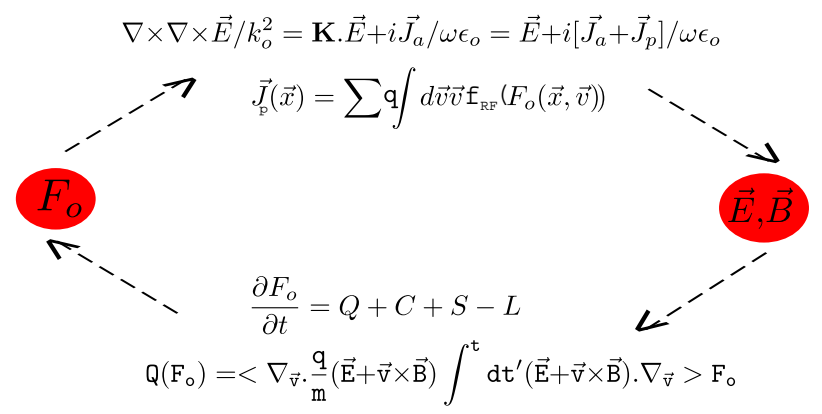

Figure 1: Schematic representation of self-consistent treatment of particle heating by electromagnetic waves.

\section{WAVE DYNAMICS AND RAY TRACING}

Lacking powerful computers allowing to solve the underlying equations truthfully, plasma heating was historically studied by making simplifications. The geometric optics or ray tracing method is a typical example. Its appealing simplicity results from the fact that 
it decouples the coexisting branches of the dispersion equation and traces their characteristics independently. As it traces the evolution of a wave, it is a powerful technique for getting insight in the details of the waveparticle interaction. Ray tracing is based on the WKB assumption that the electric field can be written in the form

$$
\vec{E} \propto \exp \left[\sum_{n=0}^{\infty} \delta^{n-1} S_{n}(\vec{x})\right]
$$

where $\delta$ is a small parameter and the $S_{n}$ vary slowly as a function of $\vec{x}$. Consider the simple 1-dimensional equation

$$
\mathrm{E} "+k^{2}(x) \mathrm{E}=0
$$

where $\mathbf{E}$ is some electric field component and ${ }^{\prime}=d / d x$. When $k^{2}$ is constant, $\mathrm{E}$ describes plane waves propagating in the $\mathrm{x}$-direction: $\mathrm{E}=\mathrm{E}_{o} \exp [ \pm i k x]$. When $k$ varies slowly as a function of $x$, a solution of the above wave equation in the form of the proposed WKB expression can be sought. Assuming $k$ is of order $1 / \delta$ and grouping the terms of like order in $\delta$ results in a system of equations for the $S_{n} \quad[6]$ :

$$
\begin{array}{r}
\left(S_{o}^{\prime} / \delta\right)^{2}+k^{2}(x)=0 ; 2 S_{o}^{\prime} S_{1}^{\prime}+S_{o} "=0 \\
2 S_{o}^{\prime} S_{n}^{\prime}+S_{n-1} "+\sum_{j=1}^{n-1} S_{j}^{\prime} S_{n-j}^{\prime}=0 ; n \geq 2
\end{array}
$$

Solving the first 2 equations above yields $E_{ \pm} \propto$ $\exp \left[ \pm i \int d x k(x)\right] / k^{1 / 2}$. The WKB version of the conservation law associated with Eq.(1) can be written $(\ln P)^{\prime}=-2 \operatorname{Im}(k)$ where $P=\operatorname{Im}\left(\mathrm{E} * \mathrm{E}^{\prime}\right)=\operatorname{Re}(k)|\mathrm{E}|^{2}$.

Reinserting Eqs.(3-5) into Eq.(1) shows that the WKB solution is a good approximation if $\mid 3 / 4\left(k^{\prime} / k\right)^{2}-$ $1 / 2 k "|k|<<\left|k^{2}\right|$ i.e. that the WKB ordering is justified when short wavelength waves are studied but that it breaks down near cutoffs $(k=0)$ and resonances or confluences $\left(k^{\prime}=\infty\right)$.

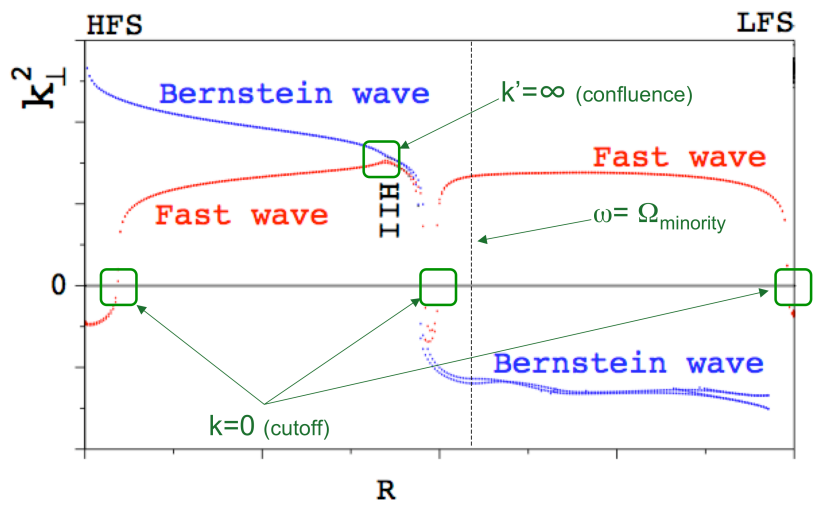

Figure 2: Dispersion plot with locations at which WKB breaks down.
Ray tracing is the multidimensional equivalent of the above scheme. Using the geometric optics definition $\vec{k}=\nabla \psi$ of the wave vector ( $\psi$ being the wave phase), the lowest order equation is the Fourier transformed wave equation for a homogeneous medium,

$$
\mathbf{G} \cdot \vec{E}_{o}=\vec{k} \times \vec{k} \times \vec{E}_{o}+k_{o}^{2} \mathbf{K} \cdot \vec{E}_{o}=\overrightarrow{0} .
$$

Nontrivial solutions exist when the dispersion equation $D=\operatorname{det}(\mathbf{G})=0$ is satisfied. The different dispersion equation roots and their eigenvectors correspond to the different decoupled waves the plasma supports. The evolution of these waves is visualized via the method of characteristics. The characteristics or rays, by definition, are given by the equations

$$
d \vec{x} / d \tau=-\partial D / \partial \vec{k} ; d \vec{k} / d \tau=+\partial D / \partial \vec{x}
$$

where $\tau$ is a variable that changes monotonically along the rays. When the dispersion is satisfied in one point on a ray, it is satisfied in all others $(\delta D=0)$. The variable $\tau$ can be linked to the physical time $t$ through the transformation $d t=\partial D / \partial \omega d \tau$. The ray equations then become

$$
d \vec{x} / d t=\partial \omega / \partial \vec{k} ; d \vec{k} / d t=+[\partial D / \partial \vec{x}] /[\partial D / \partial \omega]
$$

The first equation states that the ray's velocity is the energy propagation or group velocity $\vec{v}_{g}=\partial \omega / \partial \vec{k}$ and the second shows how the wave vector changes as a result of the background variations sensed by the ray. The ray equations can, strictly spoken, only be adopted when the plasma is lossless: as soon as dissipation is present, $D$ is complex so the ray path is no longer a real trajectory. To overcome this problem the damping is assumed to be weak such that the anti-Hermitian part $\mathbf{K}^{A}$ of the dielectric tensor is of order $\delta$ compared to the Hermitian part $\mathbf{K}^{H}$ which allows to replace $D$ by $D^{H}$ in the above equation. A power transport equation, $d \ln P / d t=[d \ln P / d \vec{x}] \cdot[d \vec{x} / d t]=-2 \operatorname{Im}(\vec{k}) \cdot \vec{v}_{g}$, generalization of the earlier mentioned conservation law, is added to the system (see e.g. [7]).

The WKB approximation is very useful when the wavelength of the waves is short w.r.t. the machine size. The fact that it breaks down near cutoffs, confluences and resonances is, however, a drawback limiting the ray tracing method's applicability. To overcome such problems one can try to solve the wave equation without making any a-priori assumptions on the dependence of $\vec{E}$ on $\vec{x}$. In the next two sections we discuss how differential equations or partial differential equations can be solved by transforming them into algebraic ones.

\section{THE FINITE DIFFERENCE METHOD}

Of all methods to tackle partial differential equations, the finite difference approach is probably the one 
that is most easily implemented. It is frequently used, both for studying wave and particle dynamics. It consists in replacing the partial differential operators in the equation and the boundary conditions by their finite difference counterparts. Doing so at each of the inner grid points and imposing the boundary conditions at the edge points, the differential problem is hereby reduced to an algebraic one that can be solved using standard matrix algebra techniques. Because the finite difference formulae only involve a small number of values of the unknown function(s) at neighbouring points, the matrices of the algebraic system are sparse. Dedicated algorithms accounting for this allow to save CPU time. Finite difference expressions for the various operators can be taken off the shelf (see e.g. [8]) or be auto-constructed from the truncated Taylor series expansion

$$
G \approx \sum_{n=0}^{N} \frac{\hat{G}_{n}\left(x-x_{o}\right)^{n}}{n !}=\sum_{j=0}^{N} \alpha_{j} G\left(x_{j}\right)
$$

where $x_{o}$ is the point for which the N-point difference scheme is constructed, and the coefficient $\hat{G}_{n}$ can be identified with the n-th derivative of $G$ at $x_{o}$ if $n \rightarrow \infty$ and if $x$ is sufficiently close to $x_{o}$. The values of the function at $x_{o}$ and at $N$ neighboring grid points are used to write down a linear system of $N+1$ equations for finite difference approximations of up to the first $N$ derivatives at $x_{o}$. Non-uniform grids are automatically accommodated for but the best performance for a given number of points is obtained using a uniform grid centered on $x_{o}$. Invoking more neighbors allows eliminating lower order contributions in the expansion: The 2-point forward scheme for the first derivative is of first order. Including the backward contribution allows to compensate the first order contribution and results in a second order accurate scheme. Doing the same for the next neighbors yields the third order scheme $\left(G_{-2}-8 G_{-1}+8 G_{+1}-G_{+2}\right) / 12 \Delta x$ for the first derivative, etc.

\section{III.A. Stability of finite difference schemes}

Lacking sufficiently general theorems, stability analysis of a numerical scheme is often done by trial and error. The diffusion equation $\Delta \psi(\vec{x}, t)=\partial \psi / \partial t$ can be solved analytically and is sufficiently simple to perform the stability analysis for various finite difference schemes. Let us start from the $1-\mathrm{D}$ version and impose Dirichlet conditions $\psi=0$ at $x=0$ and $x=1$, and $\psi=\psi_{o}(x)$ at $t=0$. Through Fourier analysis one finds $\psi=\sum_{k=1}^{\infty} \alpha_{k} \exp \left[-k^{2} t\right] \sin [k x]$, the Fourier coefficients $\alpha_{k}$ in which are those of $\psi_{o}$. Note that high $\mathrm{k}$-modes disappear fast from the exact solution when time advances. Morton and Mayers [9] examined the numerical stability of various finite difference schemes for this equation by adopting a uniform grid in both $x=j \Delta x$ and $t=n \Delta t$, and introducing the amplification factor $\lambda$ to study the evolution of the numerically obtained $k$ th Fourier mode, $\psi_{j}^{n}=\lambda^{n} \exp [i k j \Delta x]$. For the explicit forward scheme $\psi_{j}^{n+1}=\psi_{j}^{n}+\nu \delta_{x}^{2} \psi^{n}$ in which $\nu=\Delta t / \Delta x^{2}$ and $\delta_{x}^{2} \psi^{n}=\psi_{j+1}^{n}-2 \psi_{j}^{n}+\psi_{j-1}^{n}$, one finds $\lambda=1-4 \nu \sin ^{2} k \Delta x / 2$. Hence, when $\nu>0.5$ this solution numerically grows in time, although the true solution does not! The fastest growing mode is characterized by a phase jump of $\pi$ in between successive grid points. It eventually dominates the numerical solution. As the spatial grid scale is determined by the spatially fastest varying modes in the differential system, one is forced to make sufficiently small steps in time to avoid these unphysically growing solutions. To avoid having to take too small time steps, implicit rather than explicit schemes are adopted. Replacing the forward difference by a backward difference in the above we obtain the scheme

$$
-\nu \psi_{j-1}^{n+1}+(1+2 \nu) \psi_{j}^{n+1}-\nu \psi_{j+1}^{n+1}=\psi_{j}^{n}
$$

In contrast to the forward scheme, time stepping now requires the inversion of a matrix. This extra amount of work pays off, however: one finds that $\lambda$ is now of the form $\lambda=1 /\left[1+4 \nu \sin ^{2} k \Delta x / 2\right]$. Since $0 \leq|\lambda| \leq 1$ for any $\nu$, this scheme is unconditionally stable.

The above two schemes either use 3 points at the previous time level, or 3 at the new time level. A straighforward generalisation consists in using all 6 of these points i.e. in adopting the scheme $\psi_{j}^{n+1}-\psi_{j}^{n}=$ $\nu\left[\theta \delta_{x}^{2} \psi^{n+1}+(1-\theta) \delta_{n}^{2} \psi^{n}\right]$ which is known as the $\theta$ method. This scheme is conditionally stable if $\theta<1 / 2$ and unconditionally stable if $1 / 2 \leq \theta<1$. The first regime imposes that $\nu \leq 0.5 /(1-2 \theta)$ while $\nu$ is unconstrained for the second. Whereas the difference scheme is usually of first order in time, the scheme is second order accurate both in position and time when $\theta=1 / 2$. This particular scheme is due to Crank and Nicolson.

\section{III.B. Practical example}

Applying a finite difference scheme to the time dependent Fokker-Planck equation yields a system of the form

$$
\partial \vec{X} / \partial t=\mathbf{A} \cdot \vec{X}-\vec{b}
$$

in which the contributions not involving $F_{o}$ are grouped in the source term $\vec{b}$ and the values of $F_{o}$ at the grid points are stored in $\vec{X}$. When adopting the CrankNicolson method, the algebraic system takes the form

$$
\begin{gathered}
\vec{X}(t+\Delta t / 2)=\mathbf{C}^{-1} \cdot \vec{d} ; \mathbf{C}=\mathbf{1}-\Delta t / 2 \mathbf{A} ; \\
\vec{d}=[\mathbf{1}+\Delta t / 2 \mathbf{A}] \cdot \vec{X}(t-\Delta t / 2)-\Delta t \vec{b}
\end{gathered}
$$

If $\mathbf{A}$ is time independent, the $\mathbf{C}$ matrix can be inverted once and for all. The source $\vec{d}$ has to be updated at 


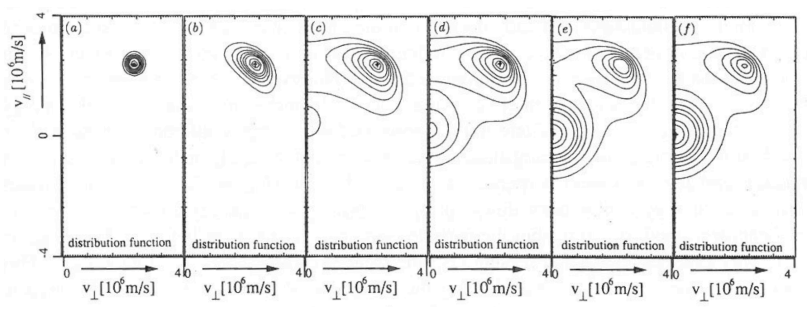

Figure 3: Time evolution of a beam population from the moment the particle source is switched on up to when the stationary state is reached, at which time the beam consists of a Maxwellian sub-population and a non-thermal sub-population centered on the source.

each time step, independent of the actual source term $\vec{b}$ being independent of time or not.

\section{THE FINITE ELEMENT METHOD}

\section{IV.A. Variational techniques}

Requiring somewhat more preparation and bookkeeping than the finite difference method but allowing a better control on the overall numerical error, the finite element method is probably the most robust numerical technique for solving differential or integro-differential problems. To discuss this technique, we will again focus on Eq.(2). The finite element technique relies on a variational principle $[10,11]$. Consider the functional

$$
\begin{array}{r}
I(\mathrm{E})=\int_{x_{1}}^{x_{2}} d x\left[-\mathrm{E}^{\prime 2}+k^{2} \mathrm{E}^{2}\right]+\hat{S} \\
\hat{S}=A_{2} \mathrm{E}^{2}\left(x_{2}\right)+B_{2} \mathrm{E}\left(x_{2}\right)-A_{1} \mathrm{E}^{2}\left(x_{1}\right)-B_{1} \mathrm{E}\left(x_{1}\right)
\end{array}
$$

Allowing for a small variation of $\mathrm{E}$, one observes that the linear perturbation of the functional is stationary for the function E obeying Eq.(2) and satisfying the boundary conditions $\mathrm{E}^{\prime}\left(x_{1,2}\right)=A_{1,2} \mathrm{E}\left(x_{1,2}\right)+B_{1,2} / 2$.

The Ritz approach to solve the equation consists in writing $\mathrm{E}$ in terms of a set of base functions $\mathrm{E}(x)=$ $\sum_{j=1}^{J} c_{j} \Theta_{j}(x)$ and imposing $I$ to be stationary for all $j$ : $\partial I / \partial c_{j}=0$. Provided the integrals involving the base functions can be evaluated, this reduces the problem of integrating the wave equation to solving the (linear) system for the $c_{j}$. Although base functions allowing to evaluate the integrals over the full domain exist (see section $\mathrm{V}$ ), one often prefers to subdivide the interval $\left[x_{1}, x_{2}\right]$ into a large number $N$ of sub-regions and to use low order polynomials with restricted range such that the integrals can be approximated e.g. using the integration method of Gauss [8]. When $J$ base functions are considered in each interval, a total of $N J$ linear equations for the $N J$ unknown coefficients is obtained by imposing all stationarity conditions. In case the grid is so dense that coefficients of the original equation hardly vary in a single finite element, their variation can be omitted altogether and the integrals only involving base functions can then be done once and for all.

The Ritz approach seems cumbersome as prior to actually solving the equation, one first needs to find the functional $I$ and derive the stationarity conditions. These 2 steps can be omitted when adopting the Galerkin approach, which consists of multiplying the differential equation with each of the base functions $\Theta_{j}$, and integrating over the domain of interest. The resulting system is again a linear system that can be solved to find the $c_{j}$. The disadvantage of this strong approach is that the $\Theta_{j}$ need to have meaningful $n$th derivatives for an $n$th order equation. Lower order polynomial base functions can be chosen when tackling the problem in its weak form i.e. after removal of the highest order E-derivative terms from the integrand by performing partial integrations. Imposing the boundary conditions via the surface term, one readily finds that the weak Galerkin approach is equivalent to the Ritz approach.
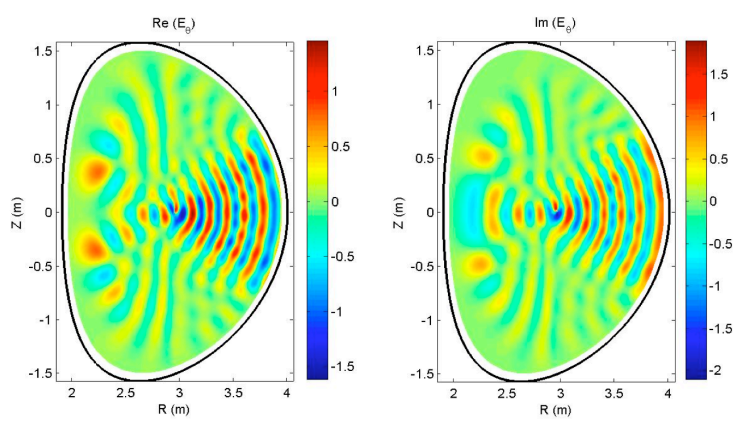

Figure 4: RF wave pattern for $(\mathrm{H})-\mathrm{D}$ heating at $3.45 \mathrm{~T}$ and 51MHZ in JET, computed using finite elements on a 2-D mesh of triangles. The RF field propagates from the antenna on the low field side to the core, where it is damped near the central $\mathrm{H}$ cyclotron layer.

\section{IV.B. Natural vs. essential boundary conditions}

Natural boundary conditions can directly be derived from the weak variational form of the equation. The strong form is obtained by multiplying the equation by a sufficiently smooth test function $G$ and integrating it over a finite interval. Partial integration is used to remove higher order derivatives from the unknown function and "transfer" them to derivatives of the test function. For a 1D differential equation of order $n, n / 2$ derivatives can be removed from the unknown. The surface terms arising from these partial integrations involve the test function itself as well as its higher order derivatives up to $n / 2-1$. Choosing a test function 
with only 1 nonzero $m$ th derivative $(0 \leq m \leq n / 2-1)$ at one of the two edges provides a set of $n / 2$ natural boundary conditions at each edge, corresponding to "fluxes" entering or leaving the domain of interest. Imposing these at the two edges provides the exact number $n$ of boundary conditions to uniquely define the solution (see e.g. [12]). Natural boundary conditions are intimately connected with the equation. Deriving the natural boundary conditions of the Laplace equation in the domain $\left(x_{1} \leq x \leq x_{2}, y_{1} \leq y \leq y_{2}, z_{1} \leq z \leq z_{2}\right)$ by multiplying the equation by a test function $G$, integrating over the volume, performing a partial integration and identifying $G=1$, one finds

$$
\left.\int d y d z \frac{\partial \psi}{\partial x}\right|_{x_{1}} ^{x_{2}}+\left.\int d x d z \frac{\partial \psi}{\partial y}\right|_{y_{1}} ^{y_{2}}+\left.\int d x d y \frac{\partial \psi}{\partial z}\right|_{z_{1}} ^{z_{2}}=0
$$

imposing that the imposed Neumann boundary conditions should be consistent with the equations i.e. that they must guarantee that the net influx balances the net outflux, no damping being present.

Boundary conditions imposed on lower order $(<$ $n / 2$ ) derivatives cannot be derived from the equation itself, and cannot be imposed via the surface term. To impose such conditions another procedure is required. One common solution is to choose the base functions in such a way that the conditions in question are automatically fulfilled. Because of their more basic nature, the second type of boundary conditions is known as essential conditions.

To solve a differential equation, we transformed it into a linear system. When the original equation is an integro-differential instead of a differential equation, exactly the same method can be used, the only difference being that also integrals of products of base functions and not just derivatives appear inside the variational integral. In that case, the linear system is generally not sparse.

\section{IV.C. Numerical pollution}

The finite element technique relies on the local approximation of the solution of an equation by a sum of simple base functions. Aside from truncation errors which automatically creep into the system, such approximations can lead to numerical pollution i.e. to the excitation of modes lacking a physical counterpart. The vacuum wave equation

$$
\begin{aligned}
\left(k_{/ /}^{2}-k_{o}^{2}\right) E_{x}+i k_{/ /} E_{z}^{\prime} & =0 \\
\left(k_{/ /}^{2}-k_{o}^{2}\right) E_{y}-E_{y}{ }^{\prime \prime} & =0 \\
i k_{/ /} E_{x}^{\prime}-k_{o}^{2} E_{z}-E_{z} " & =0
\end{aligned}
$$

suffices to demonstrate this effect [13]. In the above, it can be noted that $E_{x}$ plays a different role than $E_{y}$ or $E_{z}$ : the highest order derivative of $E_{x}$ appearing in the system is one order lower than that for $E_{y}$ and $E_{z}$. Knowing that the exact solutions of the vacuum wave equation are proportional to $\exp \left[i k_{x} x\right]$ where $k_{x}^{2}=$ $k_{o}^{2}-k_{/ /}^{2}$ we make the ansatz $\vec{E}(\vec{x})=\sum_{j} \exp \left[i k_{x} x_{j}\right] \vec{\eta}_{j}$ where the $x_{j}$ are the grid points and $\vec{\eta}_{j}$ the vectors of base functions. The discretized dispersion equation is the condition for having nontrivial solutions. Adopting the obtained equation for linear base functions, Sauter demonstrated that the physical root is well approximated by two of the numerical dispersion roots when the grid is sufficiently refined (small enough $k_{x} \Delta x$ ) but that the agreement is less good when $k_{x} \Delta x$ well exceeds 1. A third, purely numerical, root further spoils the solution. Reminding that $E_{x}$ is differentiated one time less in the wave equation, Sauter subsequently considered constants for $E_{x}$ while using linear functions for $E_{y}$ and $E_{z}$. Although one expects intuitively that such cruder procedure would lead to less accurate results, he demonstrated that - quite on the contrary the solution is now no longer polluted. One might hope that the spectral pollution problem automatically resolves itself when a more refined description (higher order polynomials as base functions) is used. It turns out that this is not the case. One finds exactly the same problem when using higher order polynomials for all components. Again, pollution can be removed choosing polynomials for $E_{x}$ which have 1 degree of freedom less than those taken for $E_{y}$ and $E_{z}$. Adding finite Larmor radius (temperature) effects to the wave equation destroys the special role $E_{x}$ plays and eliminates this particular problem of pollution.

\section{IV.D. Grid refinement techniques}

One of the peculiarities of finite elements is that is allows squeezing and stretching local finite elements, which enables describing phenomena with vastly different length scales accurately by merely reshuffling the adopted grid but without touching the structure of the local equations. Hence, rather than opting for a more complete set of base functions enabling to capture more dynamics on a fixed grid but increasing the number of local 'projection' equations, code developers often prefer to refine the grid at locations where it is needed while keeping the base functions as simple as possible. This guarantees that CPU memory is optimally used: at every location the adopted grid is just fine enough to reach the desired accuracy level, but regions not requiring a fine grid are not densely populated with grid points. Various grid refinement techniques are available in the literature, and more often than not grid generating software can be found on the www. One of the classical techniques of grid refinement is the so-called 'red-triangle/green-triangle' technique [14] adopted when subdividing the domain of interest for a 2-D problem into a set of triangles. When local 
gradients are too steep inside a triangle (red triangle), it is subdivided into 4 child-triangles born when dividing each of the 3 sides of the parent-triangle in 2 and connecting these 3 midpoints. If refinement in a neighboring triangle is required as well, the same technique is applied there while if no refinement is needed, the midpoint of the side is just connected to the opposite triangle corner (green triangle) to ensure the topology of the mesh is not changed.

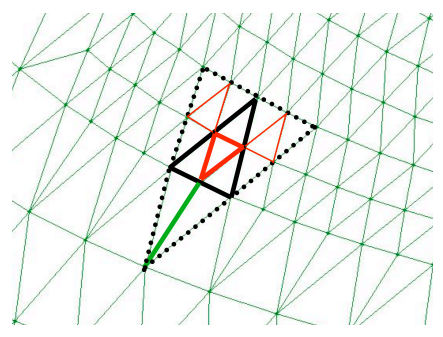

Figure 5: 'Red-green triangle' grid refinement scheme. Thick solid line: 'parent' triangle, subdivided into 4 child-triangles. Dotted lines: neighbor triangles with 'red' or 'green' subdivisions.

\section{SPECTRAL METHODS: DIRECT FOURIER RE- PRESENTATION}

As the set of exponential functions $\exp (i k x)$ is complete, any (sufficiently continuous) function of the variable $x$ can uniquely be represented by its $k$-spectrum. In a finite domain $\left[x_{o}, x_{o}+L_{x}\right]$, the coordinate $x$ can then be related to the angular variable $\theta=2 \pi x / L_{x}$ and the spectrum of modes $\mathrm{m}$ is discrete. Although any function can be represented using the exponential set with a global error that is arbitrarily small, the spectrum of non-periodic functions decays so slowly as a function of $m$ that one can wonder if the spectral method is the appropriate tool for tackling problems involving such functions. At the edge discontinuities, the series will never converge, although taking enough terms allows to find the correct value up to very close to the edge (Gibbs phenomenon). When all functions are periodic, the Gibbs phenomenon is absent and spectral representation is more appropriate. Consider again Eq.(2), $d^{2} \psi / d \theta^{2}+M^{2}(\theta) \psi=0$, for convenience rewritten in terms of the angular variable $\theta$. Finding the Fourier spectrum of $M^{2}, M^{2}(\theta)=$ $\sum_{l=-\infty}^{+\infty} M_{l}^{2} \exp [\operatorname{im} \theta]$, and projecting on the exponential base yields

$$
-m^{2} \psi_{m}+\sum_{l=-\infty}^{+\infty} M_{l}^{2} \psi_{m-l}=0
$$

for each $\mathrm{m}$ in the spectrum. Truncating the spectrum of the coefficient $M^{2}$ yields a sparse but infinite set of nontrivial equations to be solved simultaneously. For large $|m|\left(m^{2}>>M_{l}^{2}\right)$, the first term dominates the others, guaranteeing the Fourier series of $\psi$ is convergent and justifying to truncate the spectrum. Through $M^{2}$, the physics of the problem dictates the minimal number of modes to be retained in the truncated spectrum: For the simple case of a constant $M^{2}$, the above equation prescribes that $\psi_{m}$ must be zero unless $m^{2}=M^{2}$. More generally, this filtering makes that part of the physics is not captured by the model if the spectrum is truncated at a too low $m$-value (see also in the next section). A practical example is the treatment of electron (Landau + TTMP) damping in the ion cyclotron range of frequencies: the damping strength being proportional to the square of the local perpendicular wave number $k_{\perp}$, this damping is underestimated by a model that does not properly resolve the Bernstein wave mode, a mode for which $k_{\perp}$ is of the order of the inverse of the ion Larmor radius.

The spectral representation is commonly used for numerical applications posed in finite domains but is, by its nature, best suited to be adopted in wave problems. A spectral method in periodic variables is often combined with a finite element representation in the non-periodic variables.

The SciDAC project [15] gave a major thrust to RF modeling in the USA. Thanks to powerful computers and the adoption of Fourier techniques, wave problems have been solved that were off-limits before: Brambilla's TORIC has been upgraded to enable accounting for up to $10^{4}$ poloidal modes simultaneously so that the fate of short wavelength branches can now be examined in detail (see e.g. [16] ), and Jaeger's integro-differential AORSA code now solves the wave equation both for Maxwellian as for non-Maxwellian populations (see e.g. [17]).

\section{FAST FOURIER TRANSFORM \& ALIASING}

The spectral method relies on the fact that the Fourier spectra of the coefficients of the equation are known. In general the coefficients are known locally, but the spectrum is not. Finding the poloidal spectrum of functions needed to solve the $2-\mathrm{D}$ or 3 -D wave equation in tokamak geometry can be done relying on the smallness of the minor radius $a_{p}$ w.r.t. to the major radius $R_{o}$, by writing out the various terms explicitly up to a given order in the inverse aspect ratio $a_{p} / R_{o}$. This procedure soon becomes cumbersome, however, and since $a_{p}$ is not so small w.r.t. $R_{o}$, many terms in the development should be retained for a reasonable approximation. In practice, the Fourier components 
$F_{m}=\frac{1}{2 \pi} \int_{0}^{2 \pi} d \theta \exp [-i m \theta] f(\theta)$ of any needed quantity $f$ are most often evaluated numerically. Adopting a uniform grid, the Fourier integral is approximated by the sum $F_{m} \approx \frac{1}{J} \sum_{j=1}^{J} \exp [-i m j \Delta \theta] f(j \Delta \theta) ; \Delta \theta=\frac{2 \pi}{J}$ This technique is known as the fast Fourier transform (FFT). Note that the predicted value for the $m$ th Fourier component is identical to that of $m-n J$ where $n$ is any integer. This means that the above procedure artificially misrepresents high $m$-modes by their lower mode number spectrum counterparts for which $J / 2<m-n J \leq J / 2$, an effect known as aliasing (see also [18]). To avoid aliasing, the whole spectrum should fall inside the interval $]-J / 2, J / 2]$. The corresponding grid has at least 2 mesh points per wavelength for the shortest wavelength mode in the system.

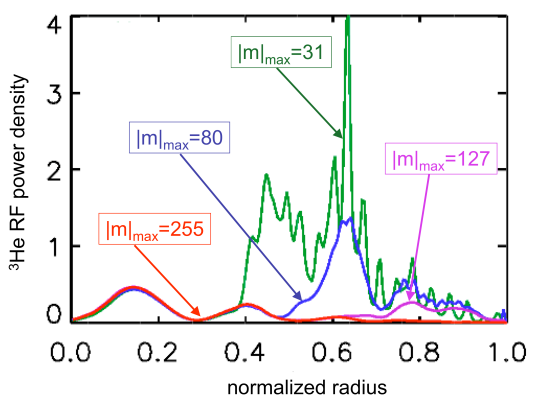

Figure 6: The importance of ensuring the full wave spectrum is sampled: power deposition profiles obtained truncating the poloidal mode spectrum at $|\mathrm{m}|=$ $31,|m|=80,|m|=127$ and $|m|=255$ (TORIC, Courtesy P. Bonoli).

\section{MONTE CARLO AND PARTICLE-IN-CELL TECHNIQUES}

Integration in multidimensional space can be done efficiently relying on the Monte Carlo technique, which in contrast to adopting a regular grid uses a set of uniformly distributed random points. Adopting this procedure, the integral of a function in hyperspace is predicted up to errors of order $N^{-1 / 2}$, where $N$ is the number of randomly generated positions, irrespective of the number of dimensions (while the accuracy of the prediction made on a uniform grid scales as $1 / N^{1 / d}$, where $d$ is the number of dimensions). In order to simultaneously solve the wave and the Fokker-Planck equations, Hedin developed the SELFO code. He upgraded the LION wave code [15] to account for the actual drift orbits of the particles and for non-Maxwellian distribution functions by locally approximating the dielectric tensor using a series of hat functions [16], and interfaced the resulting code with the FIDO Monte Carlo code [17].
The FIDO Monte Carlo method advances a large number of test particles in time $\vec{\Lambda}\left(t_{n+1}\right)=\vec{\Lambda}\left(t_{n}\right)+\Delta \vec{\Lambda}$ and accounts for wave-particle interaction and for Coulomb collisions assuming the $\vec{\Lambda}$ are stochastic variables whose expectations $E$ and co-variances $C$ can be inferred from the orbit-averaged Fokker-Planck equation:

$$
E\left[\Delta \Lambda_{i}\right]=\frac{d \mu_{i}}{d t} \Delta t ; C\left[\Delta \Lambda_{i}, \Delta \Lambda_{j}\right]=\frac{d \sigma_{i j}}{d t} \Delta t
$$

in which $<\ldots>=\int d \vec{\Lambda} F_{o} \ldots$ is the ensemble average, $\mu_{i}=<\Lambda_{i}>$ and $\sigma_{i j}=<\left(\Lambda_{i}-\mu_{i}\right)\left(\Lambda_{j}-\mu_{j}\right)>$. The Monte-Carlo operator becomes

$$
\Delta \Lambda_{i}=\frac{d \Lambda_{i}}{d t} \Delta t+\sum_{k} A^{i k} \xi_{k}(\Delta t)^{1 / 2}
$$

in which $\sum_{k} A^{i k} A^{j k}=d \sigma_{i j} / d t$ and where $\xi_{k}$ are uncorrelated stochastic variables with zero expectation value and unity variance.

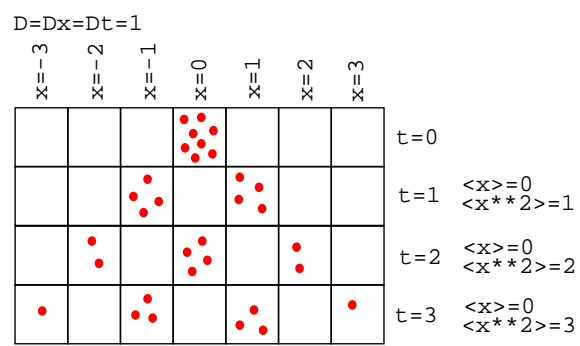

Diffusion: $\langle x * \star 2\rangle=D * t$

Figure 7: The principle of random walk / diffusion.

\section{CONSERVATION LAWS}

Conservation laws are often helpful when checking the precision of a computation. For Eq.(2) one readily sees that $S=\operatorname{Im}[E * d E / d x]$ is conserved when $k^{2}$ is real i.e. in absence of damping. When damping is present the drop in flux $S$ across the considered interval equals the integrated absorbed power $P_{a b s}=\operatorname{Im}\left(k^{2}\right)|\mathrm{E}|^{2}$. In differential form the thus obtained conservation law is of the form $\nabla \cdot \vec{S}+P_{a b s}=0$. Adopting a variational approach one can formulate the wave equation in such a conservative form i.e. in a form which readily yields this conservation law upon substituting the sufficiently smooth test function vector by the electric field (see e.g. [18]). The FokkerPlanck equation is written in the above conservative form from the start: rewriting it in variational form (see e.g. [23]), one can express the conservation of the total number of particles (test function $G=1$ ) or of the energy $\left(G=m v^{2} / 2\right)$. When the wave and FokkerPlanck models are consistent one with the other, the 
conservation laws of the 2 equations share the expression for the absorbed wave power (see e.g. [1]).

Of course, conservation laws merely allow to check the numerical accuracy of a model, but do in themselves not constitute a check on the correctness or on the applicability of the model itself. Aside from performing convergence tests to ensure the shortest scale lengths are well captured, a-posteriori checks of the assumptions underlying the derivation of an equation should be performed: it is e.g. common to adopt a truncated finite Larmor radius (FLR) expansion to include temperature effects but one rarely discards the predictions on the fate of the power carried by the short wavelength (Bernstein) modes away from the (confluence) region, notwithstanding the fact that the wave violates the starting FLR assumption ...

\section{DECIDING ON A NUMERICAL STRATEGY}

The speed and memory size of present day computers allow to pursue a high degree of realism in plasma physics models. Because of this high level of sophistication, it is crucial to be able to distinguish between actual physics and numerical artefacts. Jaun and collaborators have developed a very didactic, interactive and flexible tool to highlight the perspectives and drawbacks of various numerical schemes [18]. Existing commercial or freeware software libraries such as IMSL, HSL, NAG and NetLib allow to concentrate on physics instead of on numerics. In case no ready-made subroutines can be pulled off the shelf, softwares such as OCTAVE, MATLAB and MATHEMATICA are of great help in constructing ones own numerical schemes.

\section{REFERENCES}

1. D. VAN EESTER, Plasma Phys. Contr. Fusion 41, L23 (1999)

2. P.U. LAMALLE Plasma Phys. Contr. Fusion 39, 1409 (1997)

3. R. KOCH, Phys.Lett.A 157, 399 (1991)

4. D. VAN EESTER, Plasma Phys. Contr. Fusion 351309 (1993)

5. D. VAN EESTER, Journal of Plasma Physics 65 407 (2001) and the references therein

6. C. BENDER and S. ORSZAG, Advanced mathematical methods for scientists and engineers, Mc Graw Hill, New York (1978).

7. V.P. BHATNAGAR et al., Nuclear Fusion 24, 955 (1984)
8. M. ABRAMOWITZ, I.A. STEGUN, Handbook of Mathematical Functions, Dover, New York (1972)

9. K.W. MORTON, D.F. MAYERS, Numerical Solution of Partial Differential Equations, CUP, Cambridge (1994)

10. G. STRANG and G.J. FIX, An Analysis of the Finite Element Method, Prentice Hall, Englewood Cliffs (1973).

11. R. GRUBER and J. RAPPAZ, Finite Element Methods in Linear Ideal Magnetohydrodynamics, Springer-Verlag, New York (1985).

12. D. VAN EESTER, R. KOCH, Plasma Phys. Contr. Fusion 43, 1 (2001)

13. O. SAUTER, Nonlocal Analyses of Electrostatic and Electromagnetic Waves in Hot, Magnetized, Nonuniform, Bounded Plasmas, LRP457/92, Ecole Polytechnique Fédérale de Lausanne (1992).

14. G. HEBER, R. BISWAS, G.R. GAO, Self-Avoiding Walks over Two-Dimensional Adaptive Unstructured Grids, CAPSL Technical Memo 21, University of Delaware (1998)

15. D.B. BATCHELOR, Integrated Simulation of $\mathrm{Fu}-$ sion Plasmas, Physics Today, 02/05, 35 (2005)

16. J.C. WRIGHT et al., Phys. Plasmas 11, 2473 (2004)

17. E.F. JAEGER et al., Phys. Plasmas 13, 056101 (2006)

18. O. JAUN, J. HEDIN, T. JOHNSON, Numerical methods for partial differential equations: an overview and applications, Report TRITA-ALF-1999-05, Royal Institute of Technology, Stockholm (1999-2000); downloadable from http://www.lifelong-learners.com/pde

19. L. VILLARD et al., Comput. Phys. Rep. 4, 95 (1986)

20. J. HEDIN, Ion Cyclotron Heating in Toroidal Plasmas, Ph.D. thesis, Royal Institute of Technology, Stockholm (2001)

21. J. CARLSSON, ICRH Heating and Current Drive in Tokamaks, Ph.D. thesis, Royal Institute of Technology ISBN 97-7107-237-7 (1998)

22. D. VAN EESTER, R. KOCH, Plasma Phys. Contr. Fusion 40, 1949 (1998)

23. D. VAN EESTER Proc. 7th Eur. Fus. Theory Conf., Julich 1, 283 (1997) 